\title{
Leveraging Contact Effects for Field-Effect Transistor Technologies with Reduced Complexity and Superior Current Uniformity
}

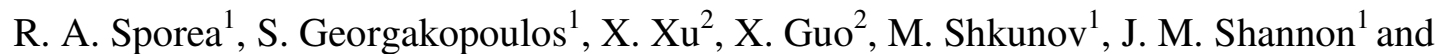 \\ S. R. P. Silva ${ }^{1}$ \\ ${ }^{1}$ Advanced Technology Institute, University of Surrey, Guildford GU2 7XH, U.K. \\ ${ }^{2}$ Department of Electronic Engineering, Shanghai Jiao Tong University, 800 Dongchuan Rd, \\ Shanghai 200240, P. R. China
}

\begin{abstract}
In order to achieve high performance, the design of devices for large-area electronics needs to be optimized despite material or fabrication shortcomings. In numerous emerging technologies thin-film transistor (TFT) performance is hindered by contact effects. Here, we show that contact effects can be used constructively to create devices with performance characteristics unachievable by conventional transistor designs. Source-gated transistors (SGTs) are not designed with increasing transistor speed, mobility or sub-threshold slope in mind, but rather with improving certain aspects critical for real-world large area electronics such as stability, uniformity, power efficiency and gain. SGTs can achieve considerably lower saturation voltage and power dissipation compared to conventional devices driven at the same current; higher output impedance for over two orders of magnitude higher intrinsic gain; improved bias stress stability in amorphous materials; higher resilience to processing variations; current virtually independent of source-drain gap, source-gate overlap and semiconductor thickness variations. Applications such as amplifiers and drivers for sensors and actuators, low cost large area analog or digital circuits could greatly benefit from incorporating the SGT architecture.
\end{abstract}

\section{INTRODUCTION}

Contact effects in thin-film transistors are currently being investigated theoretically [1] as well as experimentally [1-5]. In general these effects are responsible for a degradation in device performance mainly through a reduction in current and switching speed.

Here we show that contact effects can be used to engineer devices which have substantially better performance in some respects than the conventional TFT device architecture. The source-gated transistor (SGT) is a variation of the standard TFT transistor with what has been previously described [6] as an "extreme example" of a contact effect. SGTs are not designed with increasing transistor speed, mobility or sub-threshold slope in mind, but rather with improving certain aspects critical for real-world large area electronics such as stability, uniformity, power efficiency and gain. 
Despite the fact that the SGT architecture differs only subtly from conventional TFT, its operation is governed by a source potential barrier, which is the dominant means of controlling the current, and SGT operation is altogether different. For a transistor to be an SGT: its source needs to comprise a potential barrier; the electrode structure has to be staggered (source and gate on opposite sides of the semiconductor); and we have to be able to deplete the semiconductor layer over its whole thickness during operation.

The SGT has been studied extensively by our group, Lindner and collaborators [7] and more recently in $[1,3,8]$. Similar yet unintended behavior is observed in part in Schottky contact VLSI MOSFETs [4] where the conduction mechanism across the source barrier is seen to change in a similar fashion to what is observed in thin-film SGTs.

\section{THEORY}

The source-gated transistor (SGT) has a similar structure to a conventional staggered electrode TFT. By deliberately engineering a potential barrier at the source contact and ensuring that the gate and source electrodes overlap (Figure 1) we can control the current through the device by modulating the source barrier using the gate potential in the following way: a small drain voltage reverse biases the source barrier and depletes the semiconductor underneath. When the depletion extends across the whole semiconductor layer, the electric field generated by the gate voltage can penetrate to the source contact and modulate the effective height of the barrier and implicitly the current through the device. This is the high-field operating mode of the SGT. This mode of operation has been studied and reported in amorphous silicon $[9,10]$ and polysilicon thin [11] films and in silicon nanowire TFTs [12].

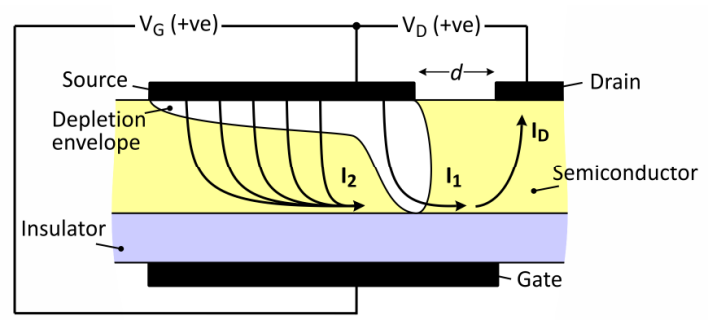

Figure 1. Schematic cross-section of a source-gated transistor (SGT) showing depletion region at the edge of the source under a small drian bias and the two current paths the relative magnitude of which gives rise to the two operating regimes.

A second mode of operation (low-field) has been outlined recently. Here, the depletion region which forms at the edge of the source closest to the drain when drain bias is applied creates a resistive current path which limits the current. Whereas in the high-field mode the majority of the current comes from a very small area at the edge of the source closest to the drain, in low-field operation, the largest proportion of the current passes through the bulk of the source contact rather than the edge area.

A seamless transition between the two modes of operation occurs and as the dominant current transport mechanism changes the transfer characteristic reflects it through a dip in 
transconductance $[4,6]$. Two-dimensional numerical simulations using Silvaco Atlas ver. 5.16.3R confirm the behavior measured in practice in a-Si:H, polysilicon and VLSI Si devices.

In both modes of operation the current saturates at a low drain voltage (Figure 2a)given by the dielectric model [10] and since the current is controlled by the source contact, the influence of drain voltage on the drain current is minimized as long as the source contact is screened from drain electric field. In other words, output characteristics can be very flat (high small signal output impedance) starting at a very low saturation voltage (Figure $2 \mathrm{~b}$ ).

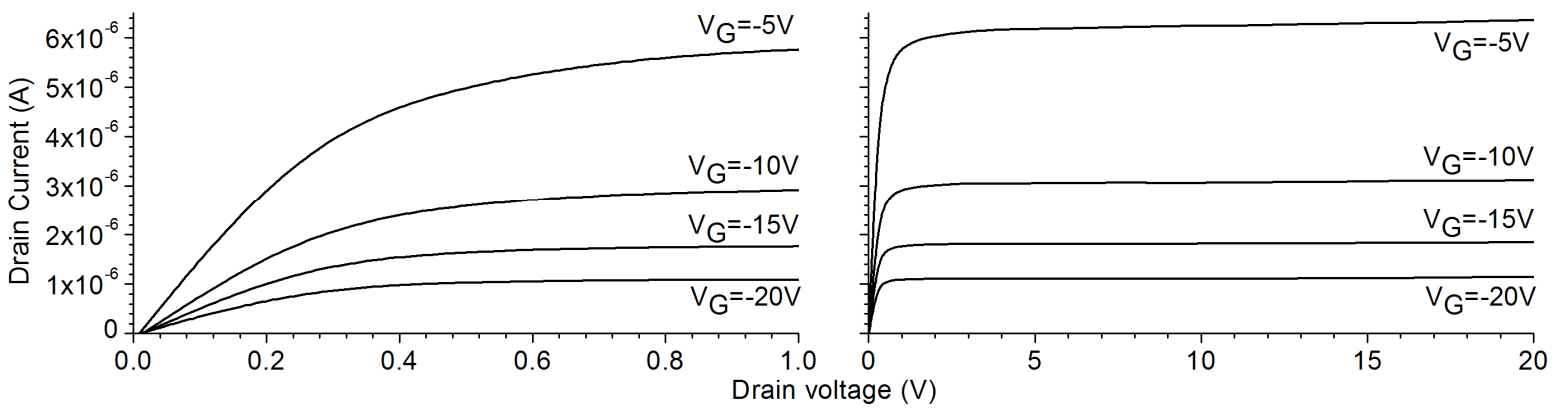

Figure 2. a) Polysilicon SGT output characteristics showing low saturation voltage. For a conventional FET, saturation voltage increases by $1 \mathrm{~V}$ for each additional $1 \mathrm{~V}$ applied to the gate, whereas for the SGT, the change in saturation voltage with drain voltage is substantially smaller [10]; b) The same characteristics plotted to high drain voltage showing flatness of the curves (low drain field dependence).

\section{DISCUSSION}

\section{$\underline{\text { SGT architecture and fabrication }}$}

SGT technology need not be more complex than conventional TFT fabrication. In fact, if steps are not taken explicitly to ensure ohmic source contact behavior and self-aligned sourcegate, it is very likely that TFT devices in a number of technologies are SGT to start with.

There are several simple ways of realizing the source barrier. The most convenient is the Schottky metal-semiconductor contact. By changing the contact metal the barrier can be tuned to different values (Figure 3a). The effective barrier height can also be changed by low energy ion implantation in silicon technologies [11] (Figure 3b) or by molecular surface modification (Figure 3c). When using a metal which does not have a large work function mismatch to the semiconductor, a barrier can be created through ion implantation to create a bulk barrier [13] (Figure 3d).

Because of the small architectural changes, FET and SGT devices can, in principle, be incorporated in the same circuit in order to take advantage of the performance characteristics of both devices (i.e. an amplifier with a SGT input stage for high gain and a FET output stage for high driving current). 

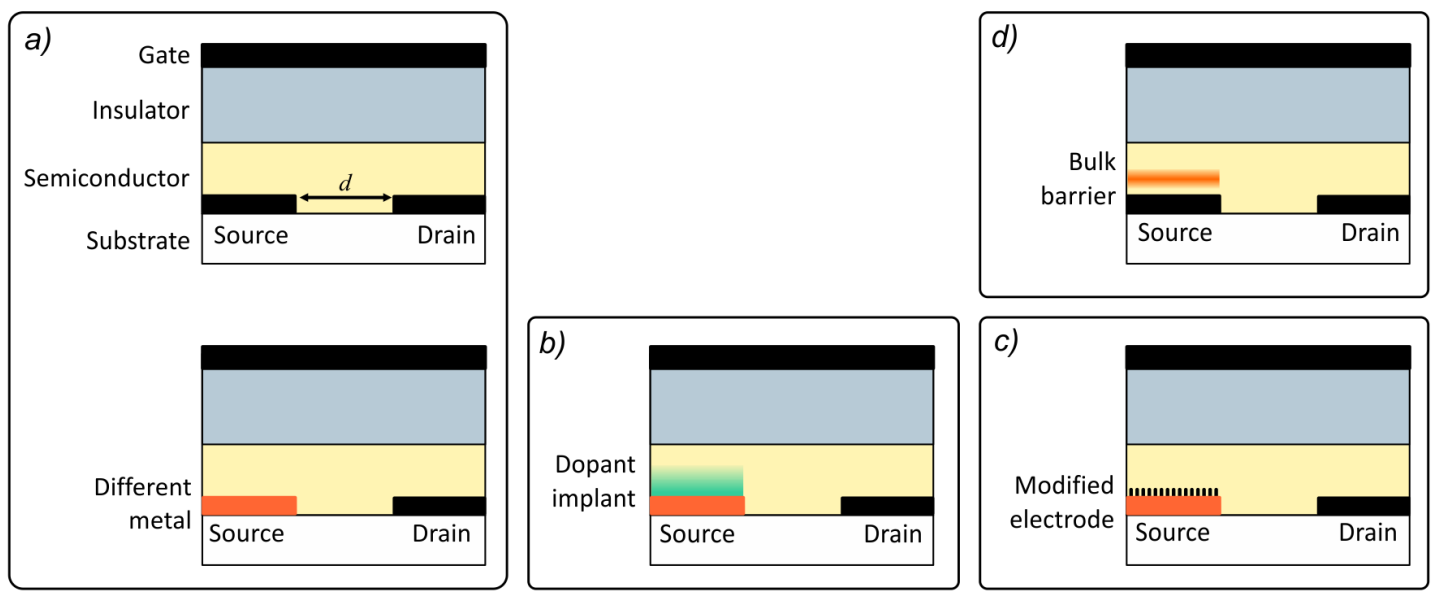

Figure 3. Starting from a staggered FET structure and as long as the semiconductor can be depleted, an SGT can be made by choosing a metal which results in a high barrier for the source electrode (a). The barrier can be tuned using implants (in silicon) (b) or by surface electrode functionalization (c). The barrier does not have to be Schottky in nature - a bulk barrier can be engineered with similar general results (d). The treatment of the drain is not important as any barrier at the drain will be forward biased and usually much more conductive than the source.

\section{Energy efficiency}

For digital circuits, power efficiency is in part given by the quality of saturation and its deterioration due to of short channel effects. The SGT excels in this respect and should be ideal for efficient, low-speed digital circuits.

In analog circuit blocks, the main parameter dictating power efficiency is the series voltage drop (source-drain voltage in the case of a transistor) at a given current. Based on the polysilicon devices we have characterized [11] we have conducted 2D numerical simulations of a single OLED pixlel driver transistor operating at a given current. We have compared SGT and FET devices in two material systems (Figure 4). Our results show significantly lower power dissipation (Figure 4a) attributed almost entirely to lower saturation voltage but also smaller layout area (Figure 4b). This second aspect is also essential for the application, as a smaller drive transistor allows a bigger pixel aperture and ultimately longer emissive material lifetime. We explain this geometrical benefit by considering that, in order to attain comparable output impedance in the presence of the kink effect, the FET channel needs to be very long; but a long channel reduces the current so the width of the device has to be increased. Since SGT current is controlled by the source, source-drain gap can be very small without compromising output impedance.

\section{$\underline{\text { Amplification }}$}

Through good source contact engineering [14] or operation in the low-field mode [6], the output impedance of the SGT, $Z_{O}$, can be extremely high, leading to very high intrinsic gain figures $\left(A_{V}=g_{m} \cdot Z_{O}\right)$. Indeed, we have measured values two orders of magnitude higher than in FETs of identical geometry occurring at five times lower drain voltage. This recommends SGTs for use in low-power high-gain large-area amplifiers. 


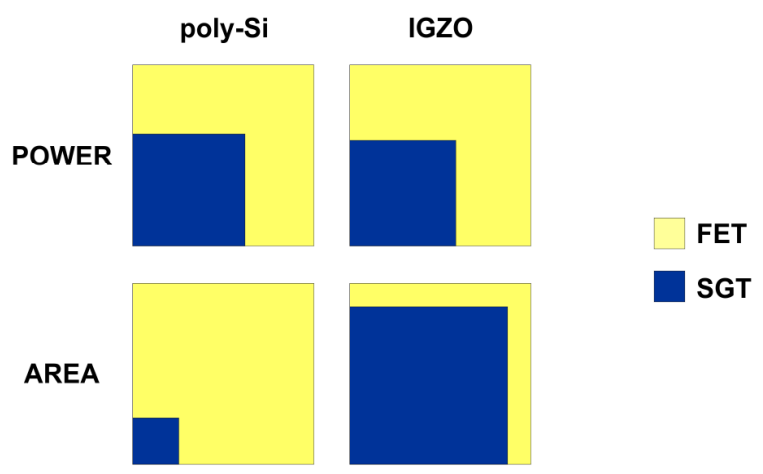

Figure 4. a) SGT power dissipation as a ratio of FET power dissipation at the same drive current; b) relative area of SGT and FET one-transistor linear drivers; for two material systems.

\section{$\underline{\text { Robust circuit fabrication in low-cost technologies }}$}

Universal to large area applications is the concern for uniformity of performance (in our case, drain current) across the whole substrate and across fabrication runs.

Intrinsic to SGT operation [6, 15], variations in source-drain gap (patterning or line edge errors) and semiconductor thickness do not impact the magnitude of drain current. Source length (or source/gate overlap - registration error) does not play a role if larger than a critical value. As long as the insulator thickness can be kept within a small tolerance (comparatively easy to achieve as the insulator is usually quite thick) and the barrier height is reproducible, SGT devices and circuits can offer consistent drain current across large areas without the need of complex built-in compensation schemes. This is particularly attractive for low-cost, low-speed applications but which require accuracy, good local amplification and power efficiency: mobile displays, remote sensors, etc.

\section{CONCLUSIONS}

Contact effects in thin-film transistors are usually deleterious and much technological and material work has been expended to eliminate them. However, we have shown that the sorucegated transistor, an "extreme example of contact effects" in a thin-film transistor can use the source contact as a means of improving several performance characteristics.

SGT architecture varies only marginally from a conventional device structure, but its operation is governed by the ability to change the conductance at the source contact using the electric field created by the gate. This leads to interesting aspects of transistor operation which are valid across a wide range of material systems and fabrication processes.

Low saturation voltage is useful in ensuring low power dissipation when transistors are used as series constant-current sources; the flat output characteristics help minimize power loss and increase amplification when SGTs are utilized as gain stages in analog amplifiers or in logic gates; the property of SGT drain current to be very weakly sensitive to changes in source-drain gap, semiconductor thickness and source length make this device ideal for robust large area applications made with low-resolution, high throughput technologies where cost is the main 
concern, as performance does not vary with changes in these parameters and there is no requirement for complex compensation techniques.

\section{ACKNOWLEDGMENTS}

The work of R. A. Sporea was sponsored by EPSRC through a PhD+ Postdoctoral Fellowship grant number EP/P503892/1 and is currently supported through the Royal Academy of Engineering Academic Research Fellowship Programme.

The work of X. Xu and X. Guo was supported in part by the National Natural Science Foundation of China under Grant 61274083, the Program for New Century Excellent Talents in University, Program for Professor of Special Appointment (Eastern Scholar) at Shanghai Institutions of Higher Learning, and Shanghai Pujiang Program (11PJ1404700).

\section{REFERENCES}

1. L. Mariucci, M. Rapisarda, A. Valletta, S. Jacob, M. Benwadi, G. Fortunato, Org. Electron. 14, 86-93 (2013).

2. H. Klauk, G.Schmid, W. Radlik, W. Weber, L. Zhou, C. D. Sheraw, J. A. Nichols, T.N. Jackson, Solid-State Electronics 47, 297-301 (2003) .

3. S. Georgakopoulos, PhD Dissertation, University of Surrey, unpublished, (2013).

4. S.-J.Choi, C.-J. Choi, J.-Y. Kim, M. Jang, and Y.-K. Choi, IEEE Trans Electron Dev., 58, 2, 427-432 (2011).

5. M. Rapisarda, A. Valletta A. Daami, S. Jacob, M. Benwadi, R. Coppard, G. Fortunato, L. Mariucci, Org. Electron. 13, 2017 (2012).

6. R. A. Sporea, J. M. Shannon and S. Ravi P. Silva, ECS PRiME 2012 Meet. Abstr. MA2012-02, 3065 (2012).

7. T. Lindner, G. Paasch, S. Scheinert, IEEE Trans Electron Dev., 51, 1, 47 - 55 (2005).

8. A. M. Ma, M. Gupta, F. Rezwana Chowdhury, M. Shen, K. Bothe, K. Shankar,Y. Tsui, D. W. Barlage, Solid-State Electronics 76, 104-108 (2012).

9. J. M. Shannon and E. G. Gerstner, IEEE Electron Dev. Lett., 24, 6, 405-407 (2003).

10. J. M. Shannon and E. G. Gerstner, Solid-State Electronics, 48, 6, 1155-1161 (2004).

11. R. A. Sporea, M. J. Trainor, N. D. Young, J. M. Shannon, S. R. P. Silva, IEEE Trans Electron Dev., 57, 10, 2434-2439 (2010).

12. C. Opoku, M. Newton, M. Shkunov, MRS Fall 2010 Abstracts, F10.3 (2010).

13. J. M. Shannon, F. Balon, Solid-State Electronics 52, 449-454 (2007) .

14. R. A. Sporea, M. J. Trainor, N. D. Young, J. M. Shannon, S. R. P. Silva, IEEE Trans Electron Dev., 59, 8, 2180 - 2186, (2012).

15. R.A. Sporea, X. Guo, J.M. Shannon, S.R.P. Silva, Proc. CAS 2009, 413-416 (2009). 